\title{
AVALIAÇÃO DA IMPLANTAÇÃO DO LABORATÓRIO DE HABILIDADES E SIMULAÇÃO: PERCEPÇÕES E VIVÊNCIAS DE DOCENTES DA GRADUAÇÃO MÉDICA
}

Gerson da Silva Ximendes, Milena Colonhese Camargo, Flávia Ferrari, Andressa Christhinie Pires, Ilana

Sobral de Luna, Airan Lobo da Costa, Lilian Francisco Arantes de Souza

Universidade do Oeste Paulista - UNOESTE, Presidente Prudente, SP. E-mail: gersonximendes@hotmail.com

\section{RESUMO}

O presente estudo teve como objetivo reconhecer as percepções e vivências de docentes, com relação ao aprendizado no laboratório de habilidades e simulação médica de uma universidade do interior paulista. Trata-se de uma pesquisa de caráter descritivo e exploratório, de natureza qualitativa. A população em estudo é constituída pelos docentes do curso de medicina de uma Universidade do interior Paulista. Os dados coletados foram avaliados e apresentados em forma de tabela e percentuais de acordo com conteúdo encontrado na avaliação, descrição e interpretação das respostas dos professores. Todos os docentes consideraram necessário o laboratório de simulações para a formação acadêmica do estudante de medicina, a principal diferença em termos comportamentais notada nos discentes após a instalação do Laboratório foi maior segurança. Conclui-se a importância do Laboratório de Habilidades e Simulação para o curso de medicina como atividade antecipatória e realística das situações que serão vivenciadas na rotina da profissão.

Palavras-chave: Laboratório, habilidade clínica, simulação, docentes de medicina, segurança.

\section{EVALUATION OF THE IMPLANTATION OF THE LABORATORY OF SKILLS AND SIMULATION: PERCEPTIONS AND EXPERIENCES OF PROFESSORS OF MEDICAL GRADUATION}

\begin{abstract}
The present study had as objective to recognize the perceptions and experiences of teachers, regarding learning in the laboratory of skills and medical simulation of a university of the interior of São Paulo. This is a descriptive and exploratory research of a qualitative nature. The population under study is made up of the professors of the medical course of a university. The collected data were evaluated and presented in table form and percentages according to the content found in the evaluation, description and interpretation of the teachers' answers. All the teachers considered necessary the laboratory of simulations for the academic formation of the student of medicine, the main difference in behavioral terms noticed in the students after the installation of the Laboratory was greater security. It concludes the importance of the Laboratory of Skills and Simulation for the medical course as an anticipatory and realistic activity of the situations that will be experienced in the routine of the profession.
\end{abstract}

Keywords: Laboratory, clinical skill, simulation, medical teachers, safety.

\section{INTRODUÇÃO}

A educação médica, sobretudo nas últimas quatro décadas, passou a se configurar como uma área multidisciplinar do conhecimento humano em intenso crescimento ${ }^{1}$. Em razão do grande número de estudos e investigações realizadas em seu âmbito, inúmeras transformações e inovações, muitas delas fundamentadas em sólidas evidências passaram a ser incorporadas nos currículos dos cursos de Medicina ${ }^{2}$. Dentre elas, encontra-se o emprego de simulações clínicas, que apresentam inúmeras 
possibilidades de aperfeiçoamento da formação profissional ${ }^{3}$.

$\mathrm{Na}$ década de 1960, o ensino médico vigente provocava insatisfações em muitas comunidades acadêmicas internacionais. Surgiram, então, algumas experiências bemsucedidas de inovações curriculares em oposição ao modelo tradicional de currículo médico. Elas tinham em comum, entre outras características: ensino centrado no aluno, adoção da metodologia do aprendizado baseado em problemas, currículo integrado, elaboração simultânea do conhecimento e das competências, diversificação dos cenários de ensino-aprendizagem e inserção precoce dos alunos em atividades profissionais. Essas inovações metodológicas e estratégicas do currículo médico propiciaram, na década seguinte, a criação de um cenário que pudesse viabilizar e sediar essas transformações: o Laboratório de Habilidades ${ }^{4}$.

O Laboratório de Habilidades representa uma alternativa de apoio pedagógico, atuando como uma atividade antecipatória das práticas de treinamento de habilidades com o paciente, adaptando o estudante ao exercício técnico e intelectual de sua futura profissão, considerando os preceitos da bioética ${ }^{4}$. No atual cenário econômico, o uso de manequins também se faz necessário, devido ao aumento dos cursos da área de saúde, da quantidade de alunos em campo de estágio e do seu impacto nos custos de aprendizagem $^{5}$. Os Estados Unidos e a Inglaterra detectaram esse problema, associado à diminuição da proporção de ambientes clínicos, o que levou à menor disponibilidade relativa de pacientes para a experiência dos estudantes ${ }^{6}$.

A clínica costuma ser o período mais estressante do curso de graduação. Esse estresse está relacionado com angústia, inexperiência, medo de cometer erros e condições de

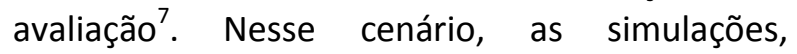
aproximam o estudante do seu cotidiano futuro, viabilizando a inserção desse indivíduo em seu contexto profissional, apresentando-se como recurso didático metodológico que permite ao discente o desenvolvimento de um olhar crítico favorecedor do aprendizado eficaz, sendo uma ferramenta capaz de impactar positivamente na redução do cenário de insegurança ${ }^{8}$.

Essa ferramenta didática revela sua importância por contribuir para a redução das situações de insegurança, controle do estresse emocional dos acadêmicos, humanizar o ensino, reforçar a interação entre os professores e estudantes e principalmente, por propiciar a redução de riscos de erros médicos e aumentar a sobrevida dos pacientes, já que a prática pode contribuir para o aprendizado a partir do erro, com o diferencial de não causar danos aos pacientes ${ }^{5}$.

Fuszard $^{9}$ defende que o conhecimento adquirido a partir dos ambientes interativos das simulações é significativo para o exercício da prática clínica por ser mantido por mais tempo que o conhecimento obtido por outros métodos didáticos. Tuoriniemi e Schott Bauer $^{10}$ acrescentam ainda que os cenários de simulação propiciam aos acadêmicos experiências psicomotoras, cognitivas e afetivas que permitem o desenvolvimento do pensamento crítico reflexivo e contribuem para a formação de novas habilidades e competências.

Considerando a existência da necessidade de treinar os profissionais de saúde as simulações a partir de materiais simuladores e equipamentos estão adquirindo destaque no conjunto das inovações educacionais, na comunicação em equipe, no desenvolvimento do pensamento cognitivo e nas ações baseadas em habilidades durante as situações de clínica sejam elas de urgência ou não ${ }^{11}$.

A experiência de simulação promove o pensamento crítico dos estudantes, contemplando cinco fatores: objetivo, fidelidade, solução do problema, apoio e feedback ${ }^{12}$. A Simulação realística é um excelente instrumento de treinamento de competências porque permite ao aprendiz atuar em ambiente controlado, sem complicadores presentes em situações reais, podendo através do feedback adequarem a técnica e a postura do mesmo preparando-o para enfrentar a realidade ${ }^{12}$.

Um trabalho realizado em um hospital do Rio de Janeiro constatou que os professores do ensino médico reforçam o potencial do ensino baseado em simulação como estratégia para desenvolvimento do processo de raciocínio clínico dos médicos em formação e ressaltam a necessidade de renovar currículos e estratégias de ensino-aprendizagem em busca de maior articulação entre teoria e prática ${ }^{13}$.

$\mathrm{O}$ objetivo deste estudo foi reconhecer as percepções e vivências de docentes do curso de medicina, com relação ao aprendizado no laboratório de habilidades e simulação clínica da Universidade do Oeste Paulista. 


\section{METODOLOGIA}

Trata-se de uma pesquisa de caráter descritivo e exploratório, de natureza quantiqualitativa. O projeto foi protocolado e aprovado pelo Comitê de Ética em Pesquisa local sob o CAAE núm. 64684317.7.0000.5515.

A população do estudo foi composta por docentes que lecionam no curso de medicina da universidade, que aceitaram participar do estudo, assinando o termo de consentimento livre e esclarecido.

Como instrumento de pesquisa foi elaborado um questionário autoaplicável baseado na literatura, com os questionamentos subdivididos em três partes, sendo: 1) Informações sócio-demográficas, 2) Informações profissionais e 3) informações sobre as perspectivas e vivencias do docente.

O procedimento de coleta e análise de dados consistiu nas seguintes etapas:

1- Explicação sobre as contribuições sociais e acadêmicas da pesquisa, durabilidade e os passos a serem percorridos;

2- Autorização e consentimento dos sujeitos que aceitaram participar através da assinatura do Termo de Consentimento Livre e Esclarecido;

3- Aplicação pelos pesquisadores, em data previamente agendada, aos docentes no local de trabalho antes do turno, ao final ou em período de intervalo, sendo os períodos diurno e vespertino em uma sala reservada, sem tempo determinado para responder o instrumento.

5- Recolhimento do instrumento logo após seu preenchimento e foram colocados em um envelope sem identificação.

6- Como critério de inclusão foi determinado: docente que leciona para os alunos do curso de medicina que tenha sido contratado a partir de 2005 (que se refere há seis anos antes da inauguração do Laboratório de habilidades e simulação) e possuíam contato prático com os alunos no hospital universitário, o critério de exclusão foi docentes contratados pela instituição após a inauguração do laboratório de habilidades e simulação, ou seja, após 2011. Foram excluídos também os que estavam de férias, licença ou que não aceitaram a participar do estudo. Assim, todos os professores da instituição que atenderam aos critérios de inclusão, além de estarem no exercício da profissão no momento da coleta de dados e aceitarem participar da pesquisa foram incluídos na amostra, totalizando 31 participantes.

$\mathrm{Na}$ interpretação procura-se atribuir um grau de significação mais amplo aos conteúdos analisados, ou seja, articula o texto descrito e analisado com os fatores que determinam suas características. Dessa forma, para realizar a interpretação é necessário ter como base a inferência e uma sólida fundamentação teórica acerca do problema investigado ${ }^{14}$.

Os dados foram analisados de forma descritiva e apresentados em tabela com frequências absolutas e percentuais das variáveis analisadas.

\section{RESULTADOS}

Participaram deste estudo 31 sujeitos. Sendo a maioria do gênero feminino, a faixa etária predominante encontrada foi no intervalo de 50 a 59 anos. Quanto ao tempo de docência, temos que de 10 a 15 anos de trabalho na instituição foi que apresentou maior frequência e que $51,61 \%$ dos docentes mantêm contato com os acadêmicos em atendimento (Tabela 1 ). 
Tabela 1. Frequência absoluta e relativa da faixa etária, gênero, tempo na docência e contato com o acadêmico dos participantes da pesquisa $(n=31)$.

\begin{tabular}{|c|c|c|}
\hline Faixa etária & Freq absoluta & Freq relativa (\%) \\
\hline 30 a 39 anos & 5 & 16,12 \\
\hline 40 a 49 anos & 10 & 32,25 \\
\hline 50 a 59 anos & 13 & 41,94 \\
\hline 60 anos ou superior & 3 & 9,68 \\
\hline \multicolumn{3}{|l|}{ Gênero } \\
\hline Feminino & 23 & 74,19 \\
\hline Masculino & 8 & 25,81 \\
\hline \multicolumn{3}{|l|}{ Tempo na docência } \\
\hline 10 a 15 anos & 17 & 54,83 \\
\hline 20 a 25 anos & 9 & 29,03 \\
\hline 25 anos ou superior & 5 & 16,12 \\
\hline \multicolumn{3}{|c|}{ Contato com o acadêmico } \\
\hline Sim & 16 & 51,61 \\
\hline Não & 15 & 48,39 \\
\hline
\end{tabular}

Quanto a percepção e vivência, os docentes foram questionados a respeito das diferenças em termos comportamentais notadas nos discentes em curso após a instalação do Laboratório de Habilidades e Simulações, se comparados com os discentes que não obtiveram esta oportunidade. Dos participantes $97 \%$ notaram diferenças e $3 \%$ não. Na especificação de quais diferenças foram notadas a mais evidente foi segurança, citada por 33\% dos professores isoladamente ou associada a postura e/ou desenvoltura. Outras diferenças notadas pelos professores foram a melhor relação prático-teórica, maior autonomia, melhor desenvolvimento da relação médico-paciente, maturidade, responsabilidade, objetividade, consciência da realidade e habilidade de conduzir situações complicadas, humanização, melhoria do raciocínio rápido, melhoria na anamnese e melhoria no exame físico.

Quando perguntados sobre o preparo do estudante ao lidar com os pacientes, $100 \%$ dos professores disseram ter visto maior preparo quando comparado a quando não havia simulação.

As características mais evidenciadas nos acadêmicos se comparados aos discentes que não passaram por treinamento simulatório, segundo os docentes foram: $94 \%$ autonomia, $48 \%$ humanização, $87 \%$ técnica, $68 \%$ preparo emocional e $3 \%$ nenhuma. Outras características citadas foram segurança, consciência e autoconhecimento.
A respeito da necessidade do laboratório de habilidades e simulação passar por atualizações, $42 \%$ dos professores acreditam que haja necessidade de atualização de materiais, instrumentos, simuladores e aparelhos e $20 \%$ acreditam que o laboratório encontra-se completo e bem atualizado. Na exemplificação de quais atualizações parecem necessárias os docentes citaram ampliação do horário de acesso; aumento do número de manequins e materiais, em especial de recém nascidos, prematuros e crianças; atualização constante de condutas e procedimentos realizados no laboratório que depois seria posto em prática no hospital universitário. Também foram citados a falta de procedimentos e materiais, porém esses equipamentos já existiam no laboratório como ausculta pulmonar e cardíaca, simulação de toracocentese e cenários de trauma e emergência.

Todos os entrevistados consideram necessário o laboratório de simulação para a formação acadêmica do estudante de medicina, sendo que $59 \%$ classificaram a melhoria no desempenho do acadêmico que passa pelo laboratório de simulação entre 76 e $100 \%$ e $41 \%$ classificaram a melhoria entre 51 e $75 \%$.

\section{DISCUSSÃO}

O presente estudo demonstra que a utilização do laboratório de simulação é crucial para o aprendizado dos discentes, a capacidade de realizar atividades em bonecos permitem que erros sejam cometidos com menor frequência. $\mathrm{O}$ 
principal método que objetiva essa melhoria no ensino é a capacidade de treino e repetição que deixam os alunos aptos a realizarem as técnicas e manobras em pessoas. Os professores que tiveram contato com os alunos antes e após a implantação do laboratório de habilidades e simulações na faculdade relataram em sua maioria que é nítido o quantos os alunos melhoraram no que tange a confiança em execução dos procedimentos médicos, a destreza no manuseio dos equipamentos e a melhora no modo de abordar os pacientes.

Segundo Mariani e Pêgo-Fernandes ${ }^{15}$, em uma análise histórica sobre estudos na área médica que utilizam a simulação como método de ensino, através de modelos mecânicos, como bonecos para ressuscitação cardiopulmonar e simuladores de realidade virtual amplamente digital, além de outros equipamentos, indicam como as principais vantagens deste método de preparo dos alunos: a capacidade de treinamento, repetição e uma melhor abordagem do preceptor durante a execução das atividades do discentes. Existem também a capacidade de diminuir o uso de animais e cadáveres na prática médica, permitindo uma redução nos conflitos éticos do treinamento na educação médica. Porém, apesar da minuciosa investigação na literatura, sobre aceleração no processo de aprendizagem ainda não tem dados definitivos que comprovam o uso dos laboratórios de habilidades possam aumentar o processo de aprendizado dos alunos.

Na pesquisa realizada por Gomez et al. ${ }^{16}$ sobre a análise do perfil de professores da área da saúde que usam a simulação como estratégia didática, também encontraram valores de variáveis utilizadas parecidas em algumas variáveis de nosso estudo, como: a prevalência dos docentes no sexo feminino; a idade dos professores a maioria se encontra na faixa etária de 50 a 60 anos, enquanto no seu estudo a maioria foi apresentada na faixa entre 25 e 49 com $70 \%$ dos entrevistados, porém $30 \%$ estava entre 51 e 60 anos de idade. Especificamente esses profissionais médicos atuam em nível de preceptores, mestres, doutores-tutores e professores associados, assim como em nossa pesquisa.

Motta e Baracat ${ }^{17}$ enfatizam que o uso de simuladores permitem uma abordagem ampla de avaliação com os alunos, através da observação direta na execução do método repassados para estes, da prática, além do grau de necessidade de cada discentes, no qual o laboratório possui um ambiente adequado e seguro pedagogicamente para o aluno, ressaltando ainda que esse tipo de avaliação pode ser somada com as avaliações escritas. Assim é possível ter uma avaliação mais global dos alunos em relação a teoria e a prática.

Para Lopez e Spirko ${ }^{18}$ o uso de laboratórios e simulações é muito importante para criação de cenários de emergências, no qual os alunos na prática real nos hospitais atuam de forma passiva na maioria das vezes observando seu preceptor atuando em casos emergências. Assim o centro de habilidades permitem com que os alunos atuem de forma ativa nestes casos de execução imediata das técnicas e procedimentos médicos. Os recursos deste processo inclui o melhor treinamento dos estudantes, avaliação mais criteriosa, simulação, preservação da autonomia do paciente. Assim eles se tornam mais preparados quando chegar na realidade da prática médica nos hospitais. Em nosso estudo, $100 \%$ dos professores avaliados frisaram o quanto o laboratório de habilidades devolveu mais confiança nos alunos para executar as técnicas e abordagem do paciente comparado quando não existia uma laboratório de habilidades e simulações.

Em países como Estados Unidos e Canadá, o uso de laboratórios de habilidades no ensino superior já é bem distribuído nas instituições, buscando sempre a expansão do ensino através de estudos com método. Quanto o Brasil, percebe-se um aumento na iniciativa de implantação dos centros de habilidades e simulações, porém vale ressaltar que o elevado custo os boneco, a expansão dos laboratórios nas faculdades dificulta 0 crescimento dos laboratórios de habilidades e simulações. Assim, encontra-se no cenário nacional uma maior adesão as instituições privados do que as públicas $^{19}$. Nas instituição no qual foi realizada a pesquisa o laboratório de habilidades e simulação, temos que foi um dos pioneiros no interior paulista, tendo uma ampla estrutura com equipamentos modernos e em uma quantidade adequada para os alunos, permitindo uma aceleração no processo de aprendizagem, através da repetição e ênfase do que os estudantes precisas melhorar.

As fragilidades encontrada no estudo foram que durante a aplicação do questionário com os professores alguns deles não relataram realizar atividades no laboratório de habilidades e simulações, apenas tinha contato com os alunos 
no hospital universitário. Assim, estes profissionais não possuíam conhecimentos de todos os equipamentos do centro de prática simulada, logo durante a pergunta: o que os laboratórios de habilidades e simulações precisava melhorar? Alguns docentes relatavam a falta de alguns equipamentos, porém estes já existiam no laboratório. Outra dificuldade encontrada no estudo foi a falta de mais estudos realizados com professores sobre o uso do laboratório de habilidades e simulações para comparar com nosso estudo.

O presente estudo demonstrou que a implantação do Laboratório de Habilidades e simulação proporcionaram resultados positivos em termos comportamentais nos discentes em curso após a instalação do laboratório quando comparados com aqueles que não tiveram tal oportunidade. Além disso, foi observado que os discentes que tiveram 0 contato com 0 laboratório estavam mais seguros, com uma postura e/ou desenvoltura melhor quando estes se encontravam com pacientes. Sendo assim conclui-se a importância do Laboratório de Habilidades e Simulação para o curso de medicina como atividade antecipatória e realística das situações que serão vivenciadas na rotina da profissão.

\section{CONFLITOS DE INTERESSE}

Os autores declaram não haver qualquer potencial conflito de interesses que possa interferir na imparcialidade deste trabalho científico.

\section{REFERÊNCIAS}

1. Kassirer JP. Redesigning graduate medical education - location and content. N Engl J Med. 1996;335(7):507-9.

DOI: https://doi.org/10.1056/NEJM199608153350709

2. Norman G. Research in medical education: three decades of progress. BMJ. 2002;324(7353):1560-2. DOI:

https://doi.org/10.1136/bmj.324.7353.1560

3. Bradley P. The history of simulation in medical education and possible future directions. Med Educ. 2006;40(3):254-62. DOI:

https://doi.org/10.1111/j.1365-

2929.2006.02394.x

4. Pezzi L, Neto SP. O laboratório de habilidades na formação médica. Cad da ABEM. 2008;4:16-
22.

5. Oliveira Gomes C, Medeiros R, Resumo GC. Processo ensino/aprendizagem no laboratório de enfermagem: visão de estudantes. Rev Gaucha Enferm. 2007;28(3):401-8.

6. Brooks N, Moriarty A, Welyczko N. Implementing simulated practice learning for nursing students. Nurs Stand. 2010;24(20):41-5. DOI: https://doi.org/10.7748/ns.24.20.41.s48

7. Beck DL, Srivastava R. Perceived level and sources of stress in baccalaureate nursing students. J Nurs Educ. 1991;30(3):127-33. DOI: https://doi.org/10.3928/0148-4834-19910301-08

8. Ferreira C. Impacto da metodologia de simulação realística, enquanto tecnologia aplicada a educação nos cursos de saúde. An Semin Tecnol Apl Educ Saúde. 2015;1(1):32-40.

9. Fuszard B, Fuszard B. Innovative teaching strategies in nursing. 2nd ed. Gaithersburg Md.: Aspen Publishers; 1995. 382p. Disponível em: http://www.worldcat.org/title/innovativeteaching-strategies-in-nursing/oclc/31709884

10. Tuoriniemi P, Schott-Baer D. Implementing a high-fidelity simulation program in a community college setting. Nurs Educ Perspect. 2008;29(2):105-9. $\quad$ DOI: https://doi.org/10.1097/00024776-20080300000012

11. Teixeira INDO, Felix JVC. Simulação como estratégia de ensino em enfermagem: revisão de literatura. Interface Comun Saúde Educ. 201;15(39):1173-84. DOI:

https://doi.org/10.1590/S141432832011005000032

12. Iglesias AG, Pazin-Filho A. Emprego de simulações no ulações no ensino e na ensino e na avaliação. Med (Ribeirão Preto). 2015;48(3):23340. DOI: https://doi.org/10.11606/issn.21767262.v48i3p233-240

13. Dourado ASS, Giannella TR. Ensino baseado em simulação na formação continuada de médicos: análise das percepções de alunos e professores de um hospital do Rio de Janeiro. Rev Bras Educ Med. 2014;38(4):460-9. DOI: https://doi.org/10.1590/S0100- 
14. Deslandes SF. Pesquisa social. São Paulo: Editora Vozes; 2011. 111p.

15. Marianil AW, Pêgo-FernandesI PM. Ensino médico: simulação e realidade virtual. Diagn Tratamento. 2012;17(2):47-8.

16. Gomez MV, Joaquim I, Vieira E, Augusto I, Neto $S$. Análise do perfil de professores da área da saúde que usam a simulação como estratégia didática. Rev Bras Educ Med. 2011.;35(2):157-62. DOI: $\quad$ https://doi.org/10.1590/S0100$\underline{5022011000200003}$

17. Motta EV, Baracat EC. Treinamento de habilidades cirúrgicas para estudantes de medicina - papel da simulação. Rev Med (São Paulo). 2018;97(1):18-2. DOI: https://doi.org/10.11606/issn.1679-

\section{6.v97i1p18-23}

18. López, JG; Spirko, LV. Simulación, herramienta para la educación médica. Salud Uninorte, Barranquilla. 2007;23(1):79-95.

19. Costa, RRO. A simulação realística como estratégica de ensino-aprendizagem em enfermagem. [Dissertação]. Mestrado em Enfermagem, Universidade Federal do Rio Grande do Norte. Natal, 2014. Disponível em: http://repositorio.ufrn.br/handle/123456789/19 550 